\title{
MRI reveals hemodynamic changes with acute maternal hyperoxygenation in human fetuses with and without congenital heart disease
}

\author{
Prashob Porayette ${ }^{1 *}$, Liqun Sun ${ }^{1}$, Edgar Jaeggi ${ }^{1}$ Lars Grosse-Wortmann', Shi-Joon Yoo ${ }^{2}$, Edward J Hickey ${ }^{4}$, \\ Steven Miller ${ }^{5}$, Christopher Macgowan ${ }^{3}$, Mike Seed ${ }^{1,2}$
}

From 18th Annual SCMR Scientific Sessions

Nice, France. 4-7 February 2015

\section{Background}

Maternal hyperoxygenation (MH) has been used for intrauterine growth restriction and proposed as a way to improve ventricular growth in the setting of congenital heart disease (CHD) [1,2]. Fetal lamb experiments reveal increases in the $\mathrm{SaO}_{2}$ of umbilical venous (UV) blood and a reduction in pulmonary vascular resistance during $\mathrm{MH}$ [3]. Doppler suggests that $\mathrm{MH}$ increases the human fetal pulmonary blood flow (PBF) [4]. The combination of fetal phase contrast (PC) MRI and MR oximetry using T2 mapping offers the potential for a comprehensive hemodynamic assessment of late gestation fetal circulation [5]. We investigated the physiologic impact of $\mathrm{MH}$ in human fetuses with and without CHD using MRI to explore the potential therapeutic benefits of chronic $\mathrm{MH}$.

\section{Methods}

We examined 17 normal human fetuses [mean gestational age (GA) of $37.3 \mathrm{wks}$; SD $\pm 1 \mathrm{wk}$ ] and 20 fetuses with CHD (mean GA of 36.2 wks; $\mathrm{SD} \pm 1 \mathrm{wk}$ ) on a $1.5 \mathrm{~T}$ system (Siemens Avanto, Erlangen, Germany) after hospital IRB approval. Flows were measured in major fetal vessels using PC MRI and indexed to fetal weight along with T2 of UV blood according to our previously published technique $[5,6]$. According to the Luz-Meiboom equation $\left[{ }^{7}\right]$ the $\mathrm{T} 2$ relaxation of blood is proportional to its $\mathrm{O} 2$ saturation. The measurements were repeated during $\mathrm{MH}(12 \mathrm{~L} / \mathrm{min}$ of $\mathrm{O} 2$ via a non-rebreather mask, $\left.\mathrm{FiO}_{2} \sim 70 \%\right)$. Results were compared using Student's $t$-test, with results with $\mathrm{p}$-value $\leq 0.05$ considered statistically significant.

${ }^{1}$ Paediatric Cardiology, The Hospital for Sick Children, Toronto, ON, Canada Full list of author information is available at the end of the article

\section{Results}

At baseline, the UV T2 was lower in CHD fetuses than in normals. Although UV T2 did not change significantly with $\mathrm{MH}$ in normals, we observed a significant increase in UV T2 in CHD fetuses with $\mathrm{MH}$ ( $\mathrm{p}=0.01$, Fig. 1, Table 1). Both groups showed a statistically significant increase in PBF during $\mathrm{MH}$ but was more dramatic in CHD fetuses $(p=0.005)$. While there was a significant reduction in ductus arteriosus (DA) flow in CHD fetuses during $\mathrm{MH}(\mathrm{p}=0.04)$, this was not present in normals. There was no significant difference in blood flow in any of the other major vessels.

\section{Conclusions}

The reason for lower $\mathrm{O} 2$ saturations in the UV of fetuses with CHD is uncertain, but may reflect abnormal placental and/or fetal cardiovascular function. The lower position of UV blood saturation on the $\mathrm{O} 2$ dissociation curve of hemoglobin may explain the higher uptake of $\mathrm{O} 2$ from maternal plasma in CHD fetuses. The expected increase in PBF with $\mathrm{MH}$ was observed in both groups. This increase in PBF also explains the lower DA flow in CHD fetuses with $\mathrm{MH}$ who had significantly higher DA flow compared to normal at baseline $(\mathrm{p}=0.002)$. This study suggests that fetal MR can be used to assess hemodynamic changes resulting from $\mathrm{MH}$ and could provide useful additional fetal monitoring when $\mathrm{MH}$ is being used for therapy.

\section{Funding}

This work was performed with support from the Labatt Family Heart Centre Innovations Fund. 


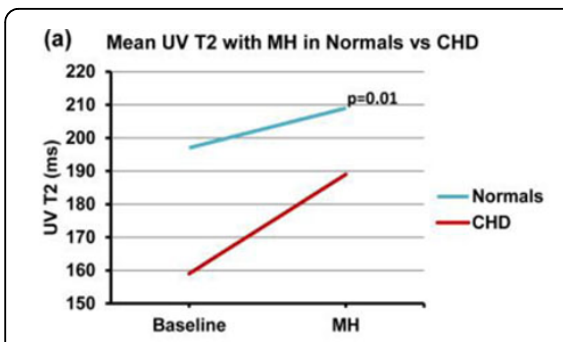

(d) Mean PBF with MH in Normals vs CHD

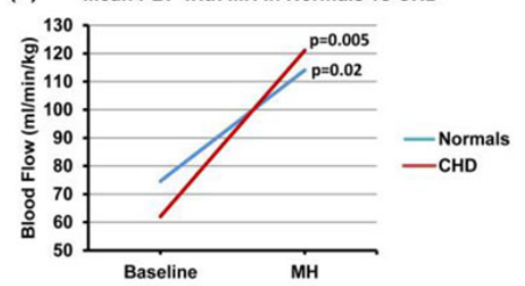

(g) Mean DA flow with MH in Normals vs CHD

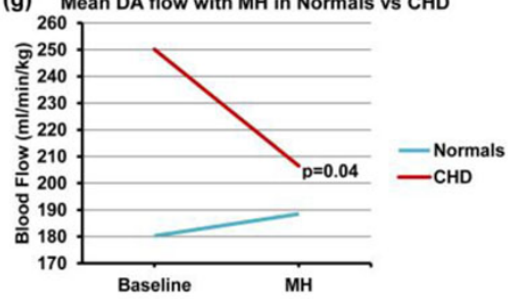

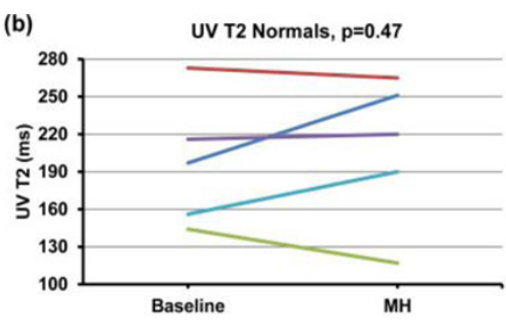

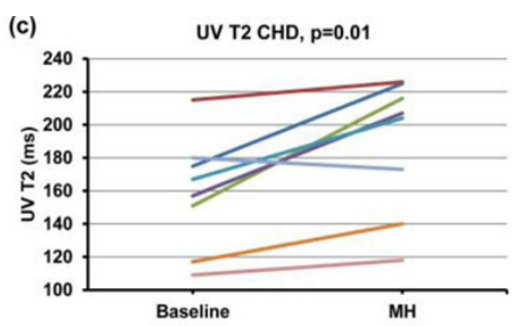

(e)
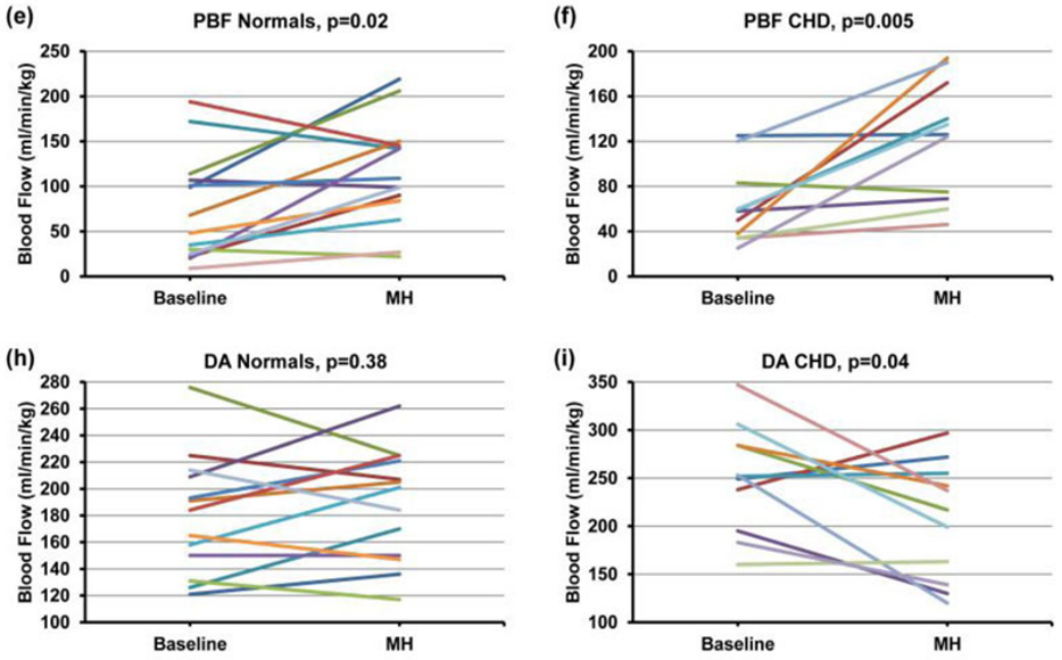

Figure 1 Physiologic impact of maternal hyperoxygenation (MH) in late gestation human fetuses with and without congenital heart disease (CHD). At baseline, the umbilical venous (UV) T2 (a) was lower in CHD fetuses than in normals. Although the UV T2 did not change significantly with $\mathrm{MH}$ in normals (b), we observed a significant increase in UV T2 in CHD fetuses with $M H$ ( $p=0.01$ ) (c). Both groups showed a statistically significant increase in pulmonary blood flow (PBF) during $\mathrm{MH}(\mathrm{d})$, which was more dramatic in fetuses with $\mathrm{CHD}(\mathrm{p}=0.005)(\mathrm{e}, \mathrm{f})$. While there was a significant reduction in ductus arteriosus (DA) flow in CHD fetuses during $M H(p=0.04)(g, i)$, this was not present in normal fetuses (h). $p \leq 0.05$ statistically significant.

Table 1 Average blood flow and T2 with maternal hyperoxygenation in human fetuses with and without congenital heart disease

\begin{tabular}{cccccccc}
\hline & Normals & & & & CHD \\
\hline & Baseline & MH & $\boldsymbol{p}$ & & Baseline & MH & $\boldsymbol{p}$ \\
\hline UV T2 (ms, $\mathbf{n = 5})$ & 197.2 & 208.6 & 0.47 & UV T2 (ms, $\mathbf{n = 8})$ & 158.88 & 188.63 & $\mathbf{0 . 0 1}$ \\
\hline PBF $(\mathbf{m l} / \mathbf{k g} / \mathbf{m i n}, \mathbf{n = 1 4})$ & 74.57 & 114.1 & $\mathbf{0 . 0 2}$ & PBF (ml/kg/min, $\mathbf{n = 1 1 )}$ & 62.36 & 121 & $\mathbf{0 . 0 0 5}$ \\
\hline DA (ml/kg/min, $\mathbf{n = 1 3 )}$ & 180.23 & 188.46 & 0.38 & DA (ml/kg/min, $\mathbf{n = 1 1 )}$ & 250.09 & 206.45 & $\mathbf{0 . 0 4}$ \\
\hline
\end{tabular}

CHD: congenital heart disease, $\mathrm{MH}$ : maternal hyperoxygenation, UV: umbilical venous, PBF: pulmonary blood flow, DA: ductus arteriosus, $\mathrm{p} \leq 0.05$ statistically significant

\section{Authors' details}

${ }^{1}$ Paediatric Cardiology, The Hospital for Sick Children, Toronto, ON, Canada.

${ }^{2}$ Diagnostic Imaging, The Hospital for Sick Children, Toronto, ON, Canada.

${ }^{3}$ Physiology \& Experimental Medicine, The Hospital for Sick Children,

Toronto, ON, Canada. ${ }^{4}$ Cardiovascular Surgery, The Hospital for Sick Children, Toronto, ON, Canada. ${ }^{5}$ Neurology, The Hospital for Sick Children, Toronto,

ON, Canada.

Published: 3 February 2015

\section{References}

1. Say, et al: Cochrane Database Syst. Rev 2003

2. Kohl: Ped Cardiol 2010.
3. Rudolph: Wiley-Blackwell 2009

4. Rasanen, et al: Circ 1998.

5. Sun, et al: ISUOG Barcelona; 2014, Abstract.

6. Prsa, et al: Circ Cardiovasc Imag 2014.

7. Luz, et al: J Chem Physics 1963.

doi:10.1186/1532-429X-17-S1-055

Cite this article as: Porayette et al:: MRI reveals hemodynamic changes with acute maternal hyperoxygenation in human fetuses with and without congenital heart disease. Journal of Cardiovascular Magnetic Resonance 2015 17(Suppl 1):O55. 